\title{
Key opinion leaders - a critical perspective
}

\section{Jose U. Scher(i) and Georg Schett (1)}

Abstract | Enormous progress has been made in the field of rheumatology in the past several decades, historically led by publicly funded academic innovators but in more recent times with much greater involvement of the pharmaceutical industry. This shift in resources has created a complex new model for reinvestment in the medical community in which the vast majority of private funds are redirected towards influencing the prescription behaviour of practitioners through 'key opinion leaders', with the main purpose of enhancing and perpetuating profit rather than innovation and critical thinking, and often at the expense of partnerships with scientists (that is, basic and translational researchers) and academic collaborations. This new episteme brings multiple opportunities to rethink approaches to sustaining long-term critical research in the field, ultimately maximizing the return on investment: scientific knowledge for the benefit of patients and society. Central to such strategies should be the rebalancing of academia-industry partnerships towards academic research and the involvement of 'innovation and knowledge leaders', rather than mostly key opinion leaders.

During the course of the past century, biomedical research has brought about previously unimaginable advances in the understanding of disease pathogenesis and therapeutics and, with these advances, a dramatic reduction in deadly infections and uncontrollable inflammatory processes. The field of rheumatology has been at the forefront of this revolution, owing in large part to the ingenuity of astute clinicians and brilliant scientists backed mostly by federal funding. Within the past three decades, another paradigm shift in the treatment of chronic immune-mediated inflammatory diseases was led by the advent of monoclonal antibody technology and other modulators of specific immune pathways. These discoveries, supported by academic innovation, were soon expanded (rather exponentially) by the pharmaceutical industry, which generated a rapid accumulation of unparalleled financial wealth and resources by private companies at a time when public funding has been stalling ${ }^{1}$.

Progress in rheumatology, as in all other fields of medicine, is dependent on the vital interaction between academic science and industry engaged in the arena. The dynamics of this relationship are complex and not always guided by the motivation to enhance knowledge and the development of improved therapeutics. The interaction between academia and industry also includes sophisticated methods that allow for the efficient spreading of opinions that can ultimately alter the prescribing patterns of physicians. In this article, we address the phenomenon of the key opinion leader (KOL), a steadily growing (in both number and influence) entity at the interface between academia and industry. Although serving as the primary nexus between companies and physicians and as a source of potentially valuable clinical information, the overall primary focus of KOLs is arguably aligned with the amplification goal of commercially driven interests. We discuss the challenges and conflicts that have emerged as a consequence of the current paradigm governing academia-industry interactions and question the pre-eminence of opinion-based leadership (that is, the KOL) at the expense of leadership based on innovation and knowledge. Finally, we present concepts and strategies to foster collaborations and expand knowledge that could lead to the discovery of novel targets for diagnostics and therapeutics in rheumatology, for the benefit of individual patients and society at large.

\section{The birth of the KOL}

In 1942, at the time when Nanna Svartz was publishing her results on the first rationally designed drug for the treatment of rheumatoid arthritis ${ }^{2}$, the communications theorist Paul Lazarsfeld was busy trying to take the science of marketing to a whole new level. Lazarsfeld was sceptical about how much the mass (that is, direct-to-consumer) media truly shaped the public's views. In the course of his research into how Ohio voters actually changed their minds during the 1940 presidential election ${ }^{3}$, Lazarsfeld discovered that human beings altered their views and preferences more because of trusted figures in their networks - or 'opinion leaders' - than because of forces such as advertising. His later work with Elihu Katz ${ }^{4}$ elaborated on their 'two-step flow of communication' theory, which suggests that opinion leaders pay close attention to the mass media and pass on their interpretation of media messages to others.

By the mid-1950s, Lazarsfeld's group had extended their argument into medicine, through a study contracted by Pfizer about the factors that influenced doctors in the USA to adopt a new drug. In this landmark study $^{5}$, the authors asked the fundamental question that continues to drive every pharmaceutical marketing operation to this day: "What were the social processes that intervened between the initial trials of the drug by a few local innovators and its final use by virtually the whole medical community?" The simple answer: the implementation of a new drug is all about promoting and expanding "the effectiveness of interpersonal relations at each stage of the diffusion process".

Thus, the concept of the KOL in medicine was born. Since then, pharmaceutical companies have continually expanded their use of the KOL model of communication. According to the Pharma Marketing Network, KOLs are physicians or non-physician scientists who are engaged by pharmaceutical companies 
to act as consultants to the companies but also to influence doctors' medical practice, including (but not limited to) their prescribing behaviour ${ }^{6}$. A catchier description of a KOL is suggested in an article in The Chronicle of Higher Education by Carl Elliott, who stated that "The KOL is a combination of celebrity spokesperson, neighbourhood gossip and the popular kid in high school"'. This latter description can be attributed to the fact that industry and KOLs often develop a kind of symbiotic relationship: industry feeds the hunger of the KOL for status and ego boosting, which motivates academic scientists to work for industry, and simultaneously KOLs support industry in the marketing of their products. It is therefore not surprising that the most desirable quality for a $\mathrm{KOL}$ is not always a scientist's knowledge or original innovative work, but rather other factors such as prescribing habits, memberships in organizations and contributions to treatment recommendations ${ }^{8}$. As outlined in TABLE 1, the fundamental function of a KOL is to act as an influencer rather than as a critical thinker; hence, the KOL acts primarily and necessarily as a marketing entity. Kimberly Elliott, an experienced former drug company sales representative, argued that "[KOLs] were sales people for us, and we would routinely measure the return on our investment, by tracking prescriptions before and after their presentations... If that speaker did not make the impact the company was looking for, then you would not invite them back" ${ }^{\prime}$. Thus, meetings, advisory boards and other events are important tools for setting up, expanding and publicizing the results of the symbiotic
industry-KOL relationship. In 2008, the rheumatologist Ted Pincus coined the term 'hotel-based medicine', suggesting that some of the myriad scientific meetings might not primarily serve the well-intentioned purpose of expanding critical knowledge but rather represent marketing vehicles for specific products $^{10}$.

\section{The two faces of the KOL}

Towards the end of his life, the painter Diego Velazquez created his masterpiece Las Meninas, one of the most celebrated and yet complex paintings of modern times $^{11}$. This enigmatic composition raises questions about reality and illusion, and the active and the passive, ultimately creating a perplexing relationship between the viewer and the figures depicted. In Las Meninas, we are unsure who is the viewer: are we watching Velazquez working or is he using us as a model? The intricate arrangement of sightlines, hiddenness and appearance in this piece of art confuses the viewer and might remind us in some ways of the KOL entity, who usually conveys commercially relevant content in an academic shroud. Conceptually, this camouflage (as in the case of the royal family depicted in Las Meninas) elevates the value and credibility of the content and represents a subliminal and efficacious strategy for reaching physician-customers, and is often complemented by other marketing strategies, including most notably dinners in high-end venues and the over-embellished industry booths found at major rheumatology meetings.

These and related approaches are commonly used in lectures (prepared by

Table 1 | Comparison of IKLs and traditional KOLs

\begin{tabular}{|lll|}
\hline Characteristic & IKL & KOL \\
\hline Function & Scientist & Influencer \\
\hline Aim & Gain of knowledge & Gain of influence \\
\hline Motivation & Hunger for knowledge & Hunger for status \\
\hline Key process & Innovation & Implementation \\
\hline Data source & Own & External \\
\hline Data handling & Data generation & Data dissemination \\
\hline Concept & To be devised/conceived & Pre-formed \\
\hline Instrument & Experiment & Steering committee \\
\hline Study type & Investigator-initiated & Industry-sponsored \\
\hline Project & Public initiative & Industry symposium \\
\hline Involvement with industry & Early- and late-stage research & Late-stage research - post asset \\
\hline Drug analogy & approval \\
\hline Newspaper section analogy & Front page & Biosimilar \\
\hline IKL, innovation and knowledge leader; KOL, key opinion leader. & \\
\hline
\end{tabular}

industry but presented by the KOL) and clinical studies (executed by companies but 'authored' by KOLs and ghostwriters) ${ }^{8}$. Concomitantly, however, the KOL

has to successfully cultivate an aura of independence. Performing such a balancing act and successfully wrapping commercial content in scientific packaging is the ultimate talent of the KOL. In a different context, these skills have been described as the 'Dr Fox effect', coined from an experiment (c. 1970) in which a lecturer's expressiveness and their being labelled an 'expert', rather than the actual content of their lecture, affected students' learning behaviour ${ }^{12}$. Hence, influencers lacking even minimal personal contributions to the matter at hand can effectively disseminate their opinions as well as the interests of their circumstantial sponsors. Today, such opinion-based influence has gained further relevance owing to the amplification power of social media. Examples from the past few years include misrepresentation of the benefits of dietary products ${ }^{13}$ as well as the unfounded hype for hydroxychloroquine as a treatment for COVID-19 (REF. ${ }^{14}$ ).

It is therefore not surprising to observe booming consulting enterprises (such as H1 or Global Vision Technology $y^{15,16}$ ) whose ultimate goal is to identify KOLs as well as to extract the critical information surrounding KOLs. For instance, these businesses claim to help "identify, analyse and apply the critical information surrounding thought leaders" ${ }^{17}$ or to "help guide marketers to optimize KOL engagements as bona fide advisers to a brand and can help shape clinical development and clinical data publication plans" ${ }^{16}$. The companies use software incorporating artificial intelligence algorithms in order to identify and engage a 'personalized' roster of KOLs that provide advocacy and feedback for a pharmaceutical company, ultimately helping to create marketing strategies for that company's products.

\section{Invest in opinion or innovation?}

The substantial interest in identifying KOLs illustrates the extent of investment by industry into the 'KOL community'. We seemingly live in times in which the dissemination of opinion is considered more desirable than investment in science-driven knowledge. Although this approach might be helpful in the short term (for example, by increasing sales and gaining market share), the mid-term and long-term consequences for rheumatology and other biomedical fields can be negative and ultimately inefficient, as industry ventures into the 
mechanistic understanding of chronic diseases and the identification of new targets are at least partially cannibalized by commercial priorities ${ }^{18}$. The monetary value of pharmaceutical industry engagement of KOLs is best illustrated by data released under the US Open Payments programme of the Physician Payments Sunshine Act, which show that in 2018 companies made payments to $\sim 627,000$ physicians totalling over US $\$ 9.35$ billion towards speaker and/or consulting fees or for the cumulative value of ownership interests ${ }^{19}$. These numbers are even more staggering when one considers that the entire NIH budget for 2018 was less than US $\$ 40$ billion $^{20}$.

Notably, despite its budget stagnating over the past two decades, the NIH continues to have an important role in spurring private success $^{21,22}$, as highlighted by the modern endogenous growth theory, which underscores the importance of 'knowledge spillovers' for long-term economic growth. In his seminal paper ${ }^{23}$, the 2018 Nobel Laureate in Economics Paul Romer argued that these knowledge spillovers (that is, when recipient entities and the economy as a whole gain material and intellectual capital that has been originally developed by others) mean that private firms (particularly pharmaceutical companies) underinvest in the production of knowledge. As a consequence, marketingdominated strategies fuelled by an excess of opinions can lead to long-term negative consequences in overall health-care outcomes. Two types of policies are aimed at ameliorating this 'market failure': patent outcomes and public funding of research. A 2019 paper examined the effects of public science on private-sector innovation in the life sciences, and came up with relevant quantitative data $^{21}$. For example, for each $\$ 10$ million invested by the NIH in a research area, there are 2.7 associated private-sector patents in that field. Similarly, it has been established that $\$ 1$ in NIH funding generates around $\$ 2.34$ in drug sales ${ }^{22}$. Therefore, and because public-sector research is crucial for private-sector innovation, it is to be expected that at least a sizeable proportion of the revenue from industry would be invested back in basic fundamental knowledge of the disease mechanism. Hence, rather than investing in opinion-multiplication by KOLs, it seems strategically wise, and sustainable in the long run, for industry to rebalance its funding towards academic research.

At present, industry contributes to $5.9 \%$ of academic research in the USA ${ }^{24}$. Research programmes aimed at addressing a specific challenge and that can have immediate applicability (that is, falling within Pasteur's quadrant or use-inspired basic research) seem to receive funding from industry more often than either purely basic or applied research ${ }^{25}$. The proportion of university research funding provided by industry can vary substantially, ranging from as low as $1 \%$ and up to $22 \%$ for a single institution ${ }^{24}$. Although the concern that industry funding might jeopardize the productivity of scientists is a valid one ${ }^{26}$, the most important channel for knowledge transfer from science to industry is in fact through the publication of research results ${ }^{27}$. Furthermore, private-public partnerships have not been shown to negatively affect academic freedom ${ }^{28}$. Critically, such initiatives enhance important indicators of innovation such as the generation of intellectual property, technology output, and numbers of jobs in high-tech sectors and new business start-ups, as well as venture capital acquisition $^{29,30}$.

\section{Rethinking the current paradigm}

Importantly, even when well-intended, the two-step flow of communication model has many inherent conflicts that necessarily lead to often blurry and difficult-to-regulate relationships between KOLs, their industry benefactors and the ultimate recipients of the primary message. The Sunshine Act and the mechanisms for disclosure and constraint of competing interests put forward by academic institutions have lessened the potential for larger conflicts. However, these stricter rules of engagement do not apply to the majority of prescribers to whom the payments are directed, or to recipients of major industry funding in university centres who 'forget' to disclose their financial conflicts ${ }^{31-33}$.

The paradox, of course, is that the authors of this Perspective are subject to the same ethical dilemmas and potential conflicts when, as academic translational scientists, we willingly interact with industry partners. The phenotypic spectrum of reactions to these challenges ranges from a puristic strategy of complete and unconditional non-engagement to a wholly laissez-faire approach without much consideration for the implications (a behaviour that inevitably generates biases and conflicts, both conscious and unconscious). One could certainly argue that the former approach is the preferable one as it has several advantages when it comes to independence and transparency. However, given the realities we have described, we believe in an intermediate, more holistic approach that has clear, well-defined rules and that is conditional on the pursuit of goals higher than personal gain.
We therefore advocate for (and practice) increased transparency in statements of competing interests (both financial and related to intellectual property) and a more rigorous clarification of funders' and sponsors' roles within the dissemination process, including presentations at scientific meetings and the publication of peer-reviewed primary research data, treatment guidelines and review articles. We also think that academicians should participate mostly - if not exclusively - in upstream scientific discussions and collaborations in which both the content and the outcomes are not responsive to (or controlled by) the sponsors (that is, advisory boards should be reserved for honest discussions about potentially available therapeutic assets and study designs but not as a vehicle to 'shape the message' of a given product). Importantly, when contributing to educational activities, the content and its presentation should be fully developed by the investigators in an independent manner and without the participation of any industry representative, whether medical or commercial.

To be clear, we are not proposing that the ultimate authority of knowledge belongs to a select group of researchers and truth can only be attained exclusively through the application of the scientific method to unsolved problems. Our overall point is not to necessarily give pre-eminence to hard science over qualitative or multicultural research, but rather to restore its value in general and particularly in the specific interactions between physicians, researchers and health-care providers. We are saying that the current paradigm will necessarily be prone to self-perpetuating bias, misinformation and a consequent lack of progress should it continue its march towards an asymmetric dialogue in which the discourse is heavily dominated by less rigorous, non-evidencebased, opinion-driven dissemination of medical content.

Consequently, and based on the outlined challenges and observations, we believe that in order to advance the field, innovative models that integrate a wide range of applied basic, clinical and translational knowledge are needed in order to synergize the many inherent strengths (that is, human and intellectual capital) available across industry and academia.

\section{Solutions and future prospects}

The French philosopher Michel Foucault published The Order of Things: An Archaeology of the Human Sciences ${ }^{34}$, in which he arrives at his central premise 
that each historical period contains certain underlying epistemological assumptions that determine what is acceptable as scientific discourse. The episteme of the current scientific period remains to be elucidated, but it is certainly one that has so far brought an unprecedented understanding of the human body and its molecular and cellular networks through an ongoing liaison between bio-mathematical modelling and biomedicine that enables the understanding of complex structures and systems. Simultaneously, however, our era is marked by complexities and ambiguities. In today's paradigm, academic institutions mutate into corporate endeavours with return-on-investment as their mantra for progress and survival, whereas for-profit pharmaceutical companies absorb some of the brightest minds in the field.

Given these new realities, the time seems ripe to reconsider the partnership between investigators and industry. Furthermore, we are convinced that interactions between pharmaceutical companies and academia can be highly innovative, lead to new concepts in disease pathogenesis, and advance the fields of rheumatology and immunology. However, such interactions need to go far beyond opinion-based spreading of information, as is often mediated by the current KOL-driven model. A truly scientific dialogue between academic scientists and industry is needed more than ever, but these interactions could (and should) be re-balanced towards innovation-driven and data-driven science. We therefore propose that innovation and knowledge - rather than opinion should constitute the foundation of the liaison between industry and academic scientists, and hence, an 'innovation and knowledge leader' (IKL), rather than a KOL, would be best suited for these interactions, ultimately leading to a long-term, mutually beneficial, innovation-driven, symbiotic relationship in pursuit of medical and scientific solutions for patients and society at large. TABLE 1 outlines the characteristics of the IKL and compares them with those of the traditional KOL.

Good examples are available in which interactions between IKLs and industry have led to outstanding advances in both immunology and rheumatology. In fact, breakthrough technological discoveries in immunology with relevance to rheumatology emerged from interdisciplinary collaborations of IKLs clustered in academialike, science-driven institutions, which were founded (and funded) by private sources. Such innovations include hybridoma-based monoclonal antibody production at The Basel Institute of Immunology (funded by Hoffman La Roche $)^{35}$, the discovery and targeting of IL-23 at DNAX (funded by Schering Plough) ${ }^{36}$ and the development of B cell-depleting strategies by targeting CD20 at Biogen IDEC ${ }^{37}$. Another notable example is the Immunology Catalyst Program designed by GlaxoSmithKline, which was dedicated to providing outstanding scientists with a 3-year sabbatical at the company's research and development hub with full access to compounds and technologies $^{38}$, or the joint venture approach illustrated by the Industry-University Cooperative Research Centers Program (IUCRC) in the USA. One can hope that similar initiatives will further proliferate, as they provide unique opportunities to study potentially interesting compounds 'on the shelf' that might be re-discovered, re-used and/or re-orientated in unexpected ways.

Furthermore, to enhance the understanding of the molecular pathogenesis of rheumatic diseases such as rheumatoid arthritis and systemic lupus erythematosus, multi-centre, multi-stakeholder, publicprivate partnerships have been established with the aim of better characterizing the molecular landscape of these diseases and of defining new treatment targets. For instance, the Accelerating Medicines Partnership (AMP) consortium in the USA is extraordinarily successful and has already served the joint interest of academia and industry to discover entirely new immune cell populations that orchestrate tissue inflammation, and has provided unbiased insights into human disease including the master regulators of the disease process ${ }^{39,40}$. Similarly, the Innovative Medicine Initiative (IMI) in Europe, which is funded by the European Union as well as partners from the European Federation of Pharmaceutical Industry (EFPIA), has made breakthroughs in understanding the molecular pathogenesis of rheumatic diseases and defining new treatment targets ${ }^{41,42}$. Without question, such partnerships would not be as fruitful if they lacked the high level of innovation that IKLs contribute to such projects. We are also convinced that, even when organizational skills are important for managing and executing such projects, these skills would not have the capacity to move the field forward and would most likely result in 'me too' projects if not paired with a critical level of innovation and knowledge (FIG. 1). Most importantly, and owing in large part to the 'honest broker' role of the $\mathrm{NIH}$ and European Union, such initiatives all but ensure that public funding and private investments from industry are mostly directed towards knowledge and innovation with little or no room for opinions.

Another productive way in which industry is currently supporting IKLs at academic institutions is by the awarding of grants through non-profit organizations such as the Rheumatology Research Foundation in the USA or the Foundation for Research in Rheumatology in Europe, as well as disease-specific non-profit organizations, such as the National Psoriasis Foundation, the Lupus Foundation of America and the Scleroderma Foundation, to name a few ${ }^{43,44}$.

Thus, a number of instruments are already in place that strategically foster industry-academia cooperation for gaining scientific knowledge. Importantly, these instruments will require mechanisms to maintain and forward-feed these collaborative efforts. Recalibrating the partnership model towards the strengthening of engagement focused on knowledge and innovation is one such approach. However, these initiatives cannot (and should not) be driven exclusively by industry; endorsement by academic centres is equally critical. Take the case of academic recognition in relation to the development of clinical trials and dissemination of their results. Currently, investigators are almost entirely credited on the basis of authorship and citation metrics. However, such measurements do not distinguish whether the conceptualization of a given study, the source of the accrued data and/or the writing of a manuscript were the product of intellectual contributions by the scientist or if they were generated by the sponsor in totality. This reward system, which intermingles industry studies with academic recognition, ultimately supports an opinion-based KOL scheme that discourages the pursuit of more laborious, albeit independent and innovative, studies. However, such a system could be modified by academia in a way that values independent science and its own intellectual property while at the same time allowing industry to present their studies in a more authentic way. This modification could be achieved through various mechanisms, including the application of metric algorithms that place higher relative weight on independent contributions for academic promotions and departmental recognition.

\section{Conclusions}

In summary, we are cognizant of the ways in which economic forces are shaping the new scientific episteme and believe that 


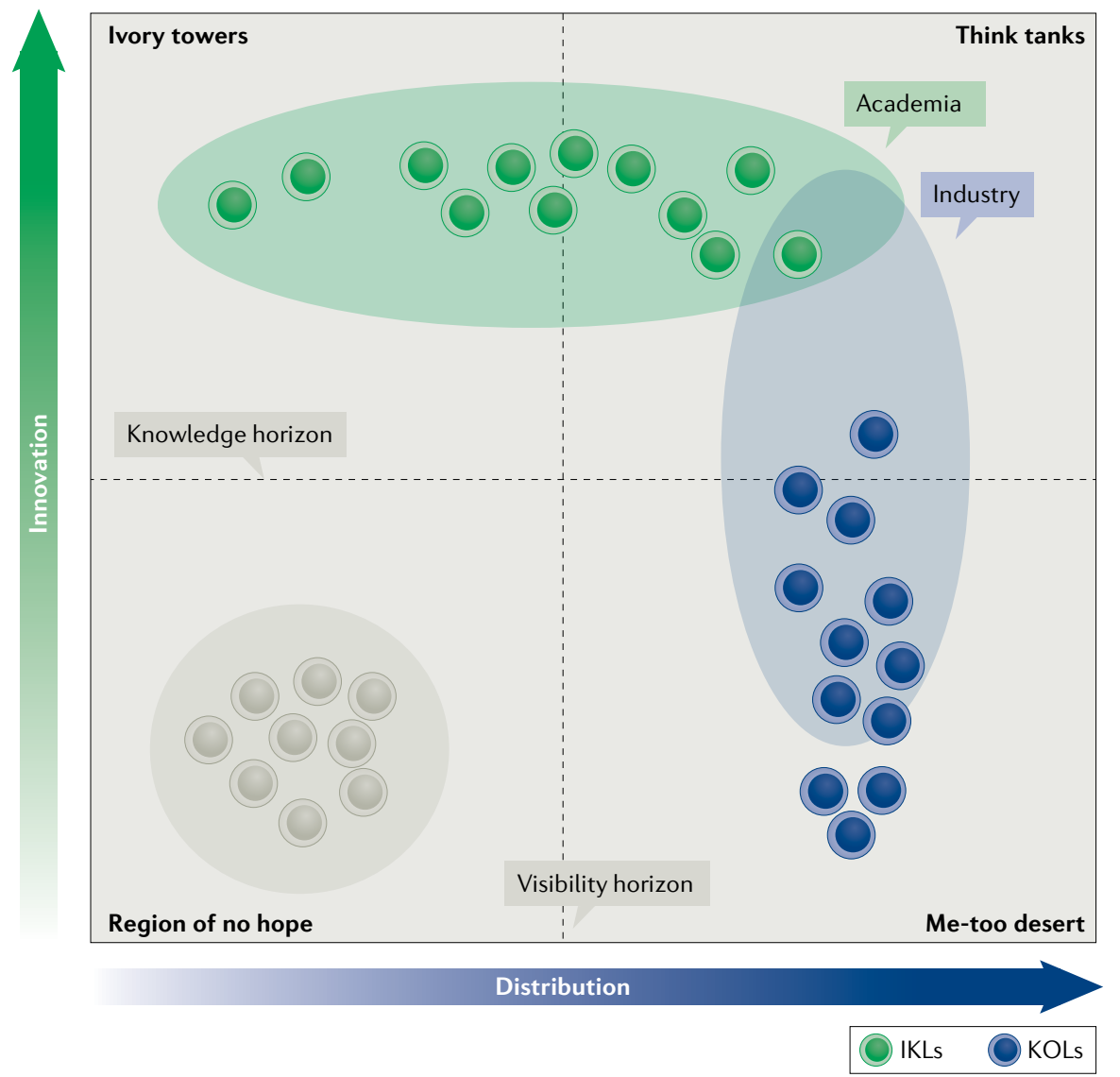

Fig. 1 Proposed Schett-Scher diagram for academia-industry collaboration. The diagram shows the relationship between two main factors that dictate the interactions between academia and industry partners: innovation and distribution. Innovation is essential for gaining critical knowledge (knowledge horizon; dashed horizontal line), and distribution is essential for gaining visbility (visbility horizon; dashed vertical line). Academic institutions are traditionally (but not exclusively) knowledge oriented, whereas industry is mostly (but not exclusively) distribution driven. Models such as think tanks involving academia and industry require high-level and comprehensive knowledge and distribution (upper right panel). Key opinion leaders (KOLs) are always distribution-oriented but not necessarily knowledge-oriented, with some of them closely situated in the 'me-too desert' space (lower right panel). Innovation and knowledge leaders (IKLs) are spread across the upper two panels according to their different levels of distribution skills. In rare extremes, IKLs in 'ivory towers' have a great deal of knowledge but poor distribution skills (upper left panel). IKLs with better distribution skills, however, are well suited to sustained innovative academia-industry partnerships and collaboration.

the authors' voices can help to reorient the conversation towards critical thinking and innovation. We are firm defendants of the benefits that the enlightenment has brought to science over the past several decades and believe that academic-industry relations should move away from an opinion-driven, commercially immersed discussion and closer to a knowledge-generating, problem-solving cooperation. Unlike facts, opinions reflect personal statements based on values and beliefs and cannot definitively be proved or disproved by objective evidence. Although this opinionated discussion is acceptable and certainly admissible in liberal democracies founded around the concepts of liberty and freedom of speech, we should be careful that they do no dominate the discourse on any field, particularly in the sciences. Otherwise, we will surely lose all sense of factual, evidence-based, critical thinking that has provided so many advances for humankind. Efforts to reinvest in knowledge and innovation, rather than merely the dissemination of opinions, will enable the development of new ideas, which in turn will refresh the field and ultimately provide the basis for the mutually beneficial, long-term sustainability of immunology, rheumatology and the pharmaceutical industry alike.

Jose U. Scher (iD ${ }^{\otimes}$ and Georg Schett $\mathrm{ID}^{2 \mathrm{凶}}$

'Department of Medicine, Division of Rheumatology, NYU Grossman School of Medicine, New York, NY, USA.
${ }^{2}$ Department of Internal Medicine, Rheumatology and Immunology, Friedrich-Alexander University Erlangen-Nuremberg and Universitätsklinikum Erlangen, Erlangen, Germany.

凶e-mail: jose.scher@nyulangone.org; Georg.Schett@ uk-erlangen.de

https://doi.org/10.1038/s41584-020-00539-1

Published online 30 November 2020

1. Burmester, G. R., Bijlsma, J. W. J., Cutolo, M \& Mclnnes, I. B. Managing rheumatic and musculoskeletal diseases - past, present and future. Nat. Rev. Rheumatol. 13, 443-448 (2017).

2. Svartz, N. Salazopyrin, a new sulfanilamide preparation. A. Therapeutic results in rheumatic polyarthritis. B. Therapeutic results in ulcerative colitis. C. Toxic manifestations in treatment with sulfanilamide preparations. Acta Med. Scand. 110, 577-598 (1942).

Lazarsfeld, P. F., Berelson, B. \& Gaudet, H. The People's Choice (Duell, Sloan \& Pearce, 1944).

4. Katz, E. \& Lazarsfeld, P. F. Personal Influence, The Part Played by People in the Flow of Mass Communications (Transaction Publishers, 1966).

5. Coleman, J., Katz, E. \& Menzel, H. The diffusion of an innovation among physicians. Sociometry 20, 253-270 (1957).

6. Pharma Marketing Network. The Pharma Marketing Clossary. https://www. pharma-mkting.com/glossary/ (2020).

7. Elliott, C. The Secret Lives of Big Pharma's 'Thought Leaders' (The Chronicle of Higher Education, 2010).

8. Sah, S. \& Fugh-Berman, A. Physicians under the influence: social psychology and industry marketing strategies. J. Law Med. Ethics 41, 665-672 (2013).

9. Moynihan, R. Key opinion leaders: independent experts or drug representatives in disguise? BMJ 336, 1402-1403 (2008)

10. Pincus, T., Bergman, M. J. \& Yazici, Y. Hotel-based medicine. J. Rheumatol. 35, 1487-1488 (2008)

11. Museo del Prado. Las Meninas https://www. museodelprado.es/en/the-collection/art-work/ las-meninas/9fdc7800-9ade-48b0-ab8b-edee94ea877f (2019).

12. Naftulin, D. H., Ware, J. E. \& Donnelly, F. A The Doctor Fox lecture: a paradigm of educational seduction. J. Med. Educ. 48, 630-635 (1973).

13. Tilburt, J. C., Allyse, M. \& Hafferty, F. W. The case of Dr. Oz: ethics, evidence, and does professional self-regulation work? AMA J. Ethics 19, 199-206 (2017).

14. Zagury-Orly, I. \& Schwartzstein, R. M. Covid-19-a reminder to reason. N. Engl. J. Med. 383, e12 (2020)

15. H1. H1 Curie an Opinion Leader Identification and Analysis Platform by $\mathrm{H} 1$ https://www.h 1 insights.com/ solutions/h 1 -curie (2020).

16. Global Vision Technology. CRM KOL Management https://www.global-visiontech.com/servicescrm-services-crm-kol-management.html (2019).

17. GlobeNewswire. H1 To Incorporate Key Opinion Leader Data In Veeva CRM Platform https://www. globenewswire.com/news-release/2018/09/10/ 1568553/0/en/H 1-To-Incorporate-Key-OpinionLeader-Data-In-Veeva-CRM-Platform.html (2018).

18. Swanson, A. Big Pharmaceutical Companies are Spending Far More on Marketing than Research (The Washington Post, 2015).

19. Policy \& Medicine. 2018 Open Payments Data Released https://www.policymed.com/2019/07/ 2018-open-payments-data-released.html (2019).

20. National Institutes of Health. Budget https://www.nih gov/about-nih/what-we-do/budget (2020).

21. Azoulay, P., Graff Zivin, J. S., Li, D. \& Sampat, B. N. Public R\&D investments and private-sector patenting: evidence from NIH funding rules. Rev. Econ. Stud. 86 117-152 (2019)

22. NIH. The National Institutes of Health (NIH): Background and Congressional Issues. https://fas.org/ sgp/crs/misc/R41705.pdf (2019).

23. Romer, P. M. The origins of endogenous growth. J. Econ. Perspect. 8, 3-22 (1994).

24. Atkinson, R. D. Information Technology \& Innovation Foundation. 2018. http://www2.itif.org/2018-industry funding-university-research.pdf (2018).

25. Stokes, D. E. Pasteur's Quadrant: Basic Science and Technological Innovation (Brookings Institution Press, 2011).

26. Hottenrott, H. \& Thorwarth, S. Industry funding of university research and scientific productivity. Kyklos 64, 534-555 (2011) 
27. Cohen, W. M., Nelson, R. R \& Walsh, J. P. Links and impacts: the influence of public research on industrial R\&D. Manag. Sci. 48, 1-23 (2002)

28. Gray, D. O. \& Walters, S. G. Managing the Industry/ University Cooperative Research Center. A Guide for Directors and Other Stakeholders (Battelle, 1998).

29. Atkinson, R. D. \& Wu, J. J. The 2017 State New Economy Index: Benchmarking Economic Transformation in the States (SSRN, 2017).

30. National Science Foundation. National Science Foundation: National Science Board, Science \& Engineering Indicators 2016 https://www.nsf.gov/ statistics/2016/nsb20161/uploads/1/13/tt08-48.pdf (2016).

31. Rosenbaum, L. Beyond moral outrage - weighing the trade-offs of COI regulation. N. Engl. J. Med. 372 2064-2068 (2015)

32. Khan, N. A., Nguyen, C. L., Khawar, T., Spencer, H. $\&$ Torralba, K. D. Association of author's financial conflict of interest with characteristics and outcome of rheumatoid arthritis randomized controlled trials. Rheumatology 58, 776-785 (2019).

33. Heneghan, C. $\&$ McCartney, M. Declaring interests and restoring trust in medicine. BMJ 367, 16236 (2019).

34. Foucault, M. The Order of Things: an Archaeology of the Human Sciences. 1966 (Vintage, 1973).

35. Köhler, G. \& Milstein, C. Continuous cultures of fused cells secreting antibody of predefined specificity. Nature 256, 495-497 (1975).

36. Oppmann, B. et al. Novel p19 protein engages IL-12p40 to form a cytokine, IL-23, with biological activities similar as well as distinct from IL-12. Immunity 13, 715-725 (2000).

37. Anderson, D. L. et al. UM Patent: Therapeutic application of chimeric antibodies to human
B lymphocyte restricted differentiation antigen for treatment of B cell lymphoma. https://experts umich. edu/details/patent/EP-1005870-A3 (2004).

38. Tkach Tuzman, K. Doctors in the house https://www biocentury.com/article/291228/gsk-is-internalizingexternal-innovation-bringing-academics-in-house (BioCentury Innovations, 2017).

39. Rao, D. A. et al. Pathologically expanded peripheral $T$ helper cell subset drives B cells in rheumatoid arthritis. Nature 542, 110-114 (2017).

40. Croft, A. P. et al. Distinct fibroblast subsets drive inflammation and damage in arthritis. Nature 570, 246-251 (2019).

41. Wohlfahrt, T. et al. PU. 1 controls fibroblast polarization and tissue fibrosis. Nature $566,344-349$ (2019).

42. Culemann, S. et al. Locally renewing resident synovial macrophages provide a protective barrier for the joint Nature 572, 670-675 (2019)

43. Davidson, A. \& Polsky, D. Sustaining the rheumatology research enterprise. Arthritis Care Res. 67, 1187 (2015).

44. Siegel, M., Shankle, L., Hwang, S. \& Ogdie, A. The 2017 National Psoriasis Foundation research symposium. J. Psoriasis Psoriatic Arthritis 3, 15-17 (2018).

Acknowledgements

J.U.S. is supported by the NIH (NIAMS R01AR074500) J.U.S. is further supported by The Riley Family Foundation The Beatriz Snyder Foundation, the Rheumatology Research Foundation and the National Psoriasis Foundation. G.S. is supported by the German Research Council (DFG: FOR2886 SFB1181), the German Ministry of Science and Education (project MASCARA), the European Union (ERC Synergy grant
4DnanoSCOPE) and the EU/EFPIA Innovative Medicines Initiative 2 (project RTCure).

\section{Author contributions}

Both authors researched data for the article and substantially contributed to discussion of content, writing and review/editing of the manuscript before submission.

\section{Competing interests}

J.U.S. declares that he has served as a consultant for Janssen, Novartis, Pfizer, Sanofi and UCB, and has received funding for investigator-initiated studies from Novartis, Sanofi and Janssen. G.S. has served as a consultant for Abbvie, BMS, Eli Lilly, Gilead, GSK Novartis, Janssen and Roche and has received funding for investigator-initiated studies from BMS, Eli Lilly, GSK, Novartis and UCB.

\section{Peer review information}

Nature Reviews Rheumatology thanks I. Adamopoulos, J. Katz and W. Lipworth for their contribution to the peer review of this work.

Publisher's note

Springer Nature remains neutral with regard to jurisdictional claims in published maps and institutional affiliations.

RELATED LINKS

Industry-University Cooperative Research Centers Program (IUCRC): https://iucrc.nsf.gov

(c) Springer Nature Limited 2020 